\title{
IDENTIFICACION MORFOLOGICA DE LAS ALETAS DE LOS PRINCIPALES TIBURONES PELAGICOS COMERCIALIZADOS EN CHILE: AZULEJO (Prionace glauca Linnaeus), MARRAJO (Isurus oxyrhinchus Rafinesque), Y TINTORERA (Lamna nasus Bonnaterre)
}

\author{
MORPHOLOGICAL IDENTIFICATION OF FINS OF THE MAIN TRADED \\ PELAGIC SHARK SPECIES IN CHILE: BLUE SHARK (Prionace glauca \\ Linnaeus), SHORTFIN MAKO (Isurus oxyrhinchus Rafinesque), AND \\ PORBEAGLE (Lamna nasus Bonnaterre).
}

\author{
Sebastián Hernández ${ }^{1,3}$, Pilar A. Haye ${ }^{1,2}$, Enzo Acuña ${ }^{1}$ \\ ${ }^{1}$ Departamento de Biología Marina, Facultad de Ciencias del Mar, Universidad Católica del Norte. Casilla 117, \\ Coquimbo, Chile.phaye@ucn.cl \\ ${ }^{2}$ Centro de Estudios Avanzados en Zonas Áridas (CEAZA), Coquimbo, Chile. \\ ${ }^{3}$ Dirección actual: School of Biological Sciences. Victoria University of Wellington, PO Box 600, Wellington \\ 6140, New Zealand. sebastian.hernandez@vuw.ac.nz
}

\begin{abstract}
RESUMEN
La carencia de registros de desembarque especie-específicos ha producido dificultades en la evaluación de stock de tiburones en Chile. Debido a que los registros son insuficientes para realizar evaluaciones pesqueras, es imperativo contar con características fidedignas para la identificación de las especies o partes de éstas (como las aletas). El presente estudio describe caracteres morfológicos para la identificación de las aletas de las tres especies más importantes de tiburones pelágicos comercializados en Chile ( $P$. glauca, I. oxyrhinchus y L. nasus) y provee de claves dicotómicas para la identificación de estas especies usando ya sea la aleta pectoral, la primera dorsal o la caudal. Los resultados revelaron que existen caracteres diagnósticos para identificar las aletas de cada una de estas especies. Ya que las especies estudiadas son las que más se comercializan, las descripciones de las aletas y las claves taxonómicas por tipo de aleta desarrolladas en este estudio prometen ser de gran utilidad para los fiscalizadores, en particular aquellos que se enfrenten a los desembarques de sacos de aletas. Además, el poder conocer tanto la cantidad como la proporción de especies comercializadas, es importante para futuras evaluaciones pesqueras y el establecimiento de planes de manejo especie-específicos.
\end{abstract}

PALABRAS ClaVE: clave taxonómica, caracteres diagnósticos, corte de aleta, finning, patrón de coloración.

\section{ABSTRACT}

The lack of species-specific landing records has produced difficulties in shark stock assessments in Chile. Given that the records are insufficient for fisheries assessments, it is imperative to have trustable morphological characteristics for the identification of species or parts of their bodies (such as fins). This study describes morphological characters useful for the identification of fins of the three main traded pelagic shark species (P. glauca, I. oxyrhinchus and L. nasus). Results showed that there are diagnostic morphological characters to identify the fins of these three species. Given that the studied species are the most traded ones, the descriptions of the fins and the taxonomic keys per fin type developed in this study should be of high use to monitor landings of shark, especially fins in sacs. The knowledge of the proportion of traded species and the quantity of each is important for future fisheries assessments and the establishment of management plans.

KEYWORDS: taxonomic key, morphological characters, finning, coloration pattern. 


\section{INTRODUCCION}

Debido al drástico aumento en las capturas de condrictios en las últimas décadas (Stevens et al. 2000; Barker \& Schlussel 2005), el manejo y la conservación de los stock pesqueros es un importante desafío para las naciones que participan en dichas pesquerías. Una de las categorías más importantes en la evaluación de stock es la estimación de la magnitud de los desembarques y la comercialización de las especies capturadas. El crecimiento de la economía Asiática durante la mitad de los años ochenta y comienzos de los noventa, provocó un aumento de 30 veces del valor por kilo de las aletas secas de tiburón (Musick et al. 2000). De esta manera, se incentivó la pesquería mundial de tiburones y consecuentemente la práctica del corte de aletas o finning. Esta consiste en la remoción de las principales aletas de interés comercial, para posteriormente descartar el resto del cuerpo vivo al océano, donde mueren por desangramiento. Algunos de los motivos esgrimidos para explicar el descarte corresponden a la baja capacidad de almacenaje de las bodegas de las embarcaciones y para evitar la descomposición de los cuerpos en éstas (Camhi et al. 1998; Stevens et al. 2000; Shivji et al. 2002).

En Chile, no hay información especieespecifica de los tiburones comercializados (Lamilla et al. 2005; Hernández et al. 2008). En el norte operan embarcaciones artesanales e industriales que capturan tiburones pelágicos como especie objetivo y de forma incidental (Lamilla et al. 2005; Hernández et al. 2008). Las pesquerías realizadas por el sector artesanal corresponden a la del pez espada (Xiphias gladius Linnaeus) y la del tiburón marrajo (Isurus oxyrhinchus Rafinesque), las cuales operan dentro de la Zona Económica Exclusiva (ZEE) entre la I ( $18^{\circ} 21^{\prime} \mathrm{S}$, $\left.70^{\circ} 22^{\prime} \mathrm{W}\right)$ y la $\mathrm{V}$ región $\left(32^{\circ} 08^{\prime} \mathrm{S}, 71^{\circ} 31^{\prime} \mathrm{W}\right)$. El sector industrial por su parte desarrolla principalmente la pesquería de pez espada, la cual realiza sus faenas de pesca principalmente fuera de la ZEE (Acuña et al. 2002; Lamilla et al. 2005; Hernández et al. 2008).

Los principales puertos de desembarque de tiburones pelágicos en el norte de Chile son Caldera $\left(27^{\circ} 03^{\prime} \mathrm{S}, 70^{\circ} 49^{\prime} \mathrm{W}\right)$ y Coquimbo $\left(29^{\circ} 57^{\prime} \mathrm{S}, 71^{\circ}\right.$ $\left.20^{\prime} \mathrm{W}\right)$. Generalmente, los pescadores desembarcan troncos y una gran cantidad de sacos de aletas, las cuales no se registran en las estadísticas oficiales del Servicio Nacional de Pesca (SERNAPESCA 1996-2006) y solamente los primeros figuran en los registros de desembarque. Sin embargo, debido a la práctica del corte de aletas, y el posterior descarte de una cantidad desconocida de troncos, la información sobre los stocks de las distintas especies se encuentra subestimada.

En Chile la práctica del corte de aleta no está regulada a pesar de la aprobación del Plan de Acción Nacional para la Conservación de Tiburones (Decreto No 198/07 del 2007). Los troncos son comercializados en el mercado nacional e internacional, mientras las aletas son exportadas exclusivamente al mercado Asiático (Lamilla et al. 2005). Las principales especies pelágicas comercializadas son el azulejo (Prionace glauca Linnaeus), el marrajo o tiburón (Isurus oxyrhinchus), y la tintorera (Lamna nasus Bonnaterre) (Lamilla et al. 2005, Hernández et al. 2008). La identificación de aletas de tiburones pelágicos en los secaderos de la zona centro-norte de Chile, sugiere que más del $80 \%$ de las que son comercializadas pertenecen a la especie Prionace glauca, mientras que el resto está compuesto por las dos especies de lámnidos: I. oxyrhinchus y L. nasus; además del pejezorro Alopias superciliosus (Lowe) (Hernández et al. 2008). Adicionalmente, se han identificado mediante el uso de técnicas moleculares al menos otras seis especies de tiburones involucradas en la comercialización de aletas: A. vulpinus (Bonnaterre), Carcharhinus obscurus (Lesueur), Galeorhinus galeus (Linnaeus), Isurus paucus (Guitart Manday), Sphyrna zygaena (Linnaeus), y S. lewini (Griffith \& Smith) (Hernández et al. 2008).

Para tener registros más detallados y fidedignos de los desembarques es necesario incorporar la identificación de las especies a las que pertenecen las aletas comercializadas, lo que es esencial para determinar la composición y proporción real de tiburones asociados al viaje de pesca (inclusive tallas de captura, a partir de factores de conversión, desarrollados por Acuña e et al. $2001 \mathrm{y}$ Hareide $e t$ al. 2007) y por ende para la evaluación de stock. En este contexto, el objetivo del presente estudio es proporcionar una herramienta morfológica fidedigna para la identificación de las aletas de las tres especies más importantes involucradas en el comercio de aletas, que son $P$. glauca, $I$. oxyrhinchus y L. nasus. 


\section{MATERIALES Y METODOS}

Se obtuvieron ejemplares de $P$. glauca $(n=15), I$. oxyrinchus $(n=15)$, y L. nasus $(n=10)$, desde naves industriales de la pesquería del pez espada ( $X$. gladius), las cuales desembarcan en el puerto de Coquimbo. Las aletas pectoral, primera dorsal y caudal, fueron removidas de los ejemplares previamente identificados a nivel de especie. Se describió la forma de las aletas, así como el patrón de coloración, presencia de estructuras características y proporciones de medidas morfométricas de las aletas (Compagno 2002) (Figura 1). Además se construyeron claves taxonómicas para la identificación de cada tipo de aleta de las tres especies consideradas en el estudio.

\section{RESULTADOS}

Descripción Morfológica De las Aletas

A continuación se presenta la descripción morfológica externa de las aletas pectoral, primera dorsal y caudal de P. glauca, I. oxyrinchus y L. nasus:

\section{Prionace glauca}

PeCtORAL: Márgenes anterior y posterior rectos (Figura 1a). El margen anterior es tres veces más largo que la base de la aleta (Tabla I). La superficie dorsal es azul oscuro con marcadas ceratotrichas (fibras de cartílago que componen la aleta internamente) (Figura 1a), mientras que la superficie ventral es completamente blanca (Figura 1b). Primera Dorsal: El margen anterior es recto y el posterior curvado en forma de "V" (Figura 1c). El borde libre es igual a la mitad de la longitud de la base (Tabla I) y es azul oscuro con marcadas ceratotrichas.

CAUDAL: El lóbulo superior es más del doble de largo que el inferior (Tabla I; Figura 1d). Es azul oscuro. El margen terminal del lóbulo superior es levemente convexo (Figura 2c). No presenta quillas laterales.

\section{Isurus oxyrhinchus}

Pectoral: Márgenes anterior y posterior triangularles isométricos y el margen posterior es notoriamente curvado (Figura 1e). El margen anterior es tres veces más corto que la base de la aleta (Tabla I). La superficie dorsal es azul oscuro (Figura 1e) y la ventral es completamente blanca con los bordes levemente oscurecidos (Figura 1f). PRIMERA DORSAL: El margen anterior es curvado y el posterior levemente convexo. El borde libre es menos de un tercio de la longitud de la base (Tabla I) y es azul oscuro en los bordes y hacia el centro es de un patrón azul-metálico (Figura 1g).

CAUdAL: La aleta tiene una notoria forma de media luna (Figura 1h). El lóbulo superior es levemente más largo que el inferior (Tabla I). Es azul oscuro con una notoria mancha blanca en la región debajo de la quilla. El margen terminal del lóbulo superior es recto (Figura 2h). Margen pos-ventral inferior es de forma recta, sin presencia de hilachas (Figura 2h). Presenta una quilla lateral a cada lado.

TABLa I. Promedio y desviación estándar de las relaciones morfométricas de las aletas pectoral, dorsal, y caudal de $P$. glauca, I. oxyrinchus y L. nasus. Siglas de nombres de medidas morfométricas según Figura 1.

TABLE I. Mean and standard deviation of the main morphometric relationships of the pectoral, dorsal and caudal fins of P. glauca, I. oxyrinchus y L. nasus. Morphometric measurement abbreviations according to Figure 1.

\begin{tabular}{|c|c|c|c|c|c|c|c|}
\hline \multirow[t]{2}{*}{ Especie } & \multirow[t]{2}{*}{$n$} & \multicolumn{2}{|c|}{ Aleta pectoral } & \multicolumn{2}{|c|}{ Aleta dorsal } & \multicolumn{2}{|c|}{ Aleta caudal } \\
\hline & & LMA & LB & LBL & LB & LMA & LMP \\
\hline P. glauca & 15 & $22,7 \pm 10,3$ & $7,4 \pm 2,9$ & $5,4 \pm 2,4$ & $10,1 \pm 4,0$ & $37,1 \pm 10,6$ & $16,6 \pm 4,9$ \\
\hline I. oxyrinchus & 15 & $20,7 \pm 4,6$ & $8,3 \pm 2,5$ & $2,7 \pm 0,7$ & $12,5 \pm 2,6$ & $26,4 \pm 6,3$ & $19,5 \pm 5,0$ \\
\hline L. nasus & 10 & $17,0 \pm 4,9$ & $6,1 \pm 2,9$ & $3,0 \pm 1,0$ & $8,0 \pm 3,7$ & $20,5 \pm 6,3$ & $14,0 \pm 4,7$ \\
\hline
\end{tabular}


Gayana 73(1), 2009

\section{Lamna nasus}

PeCTORAL: Márgenes anterior y posterior triangulares isométricos, el posterior es notoriamente curvado (Figura 1i). El margen anterior es tres veces más corto que la base de la aleta (Tabla I). La superficie dorsal es gris oscuro (Figura 1i), mientras que la ventral tiene un notorio patrón oscuro que nace desde el extremo de la aleta hasta el borde posterior interno (Figura 1j).

PRIMERA DORSAL: El margen anterior es curvado y posterior es levemente convexo. Coloración completamente grisácea. El borde libre es menos que un tercio de la longitud de la base (Tabla I) y su extremo es evidentemente blanco (Figura $1 \mathrm{k}$ ).

\section{a}
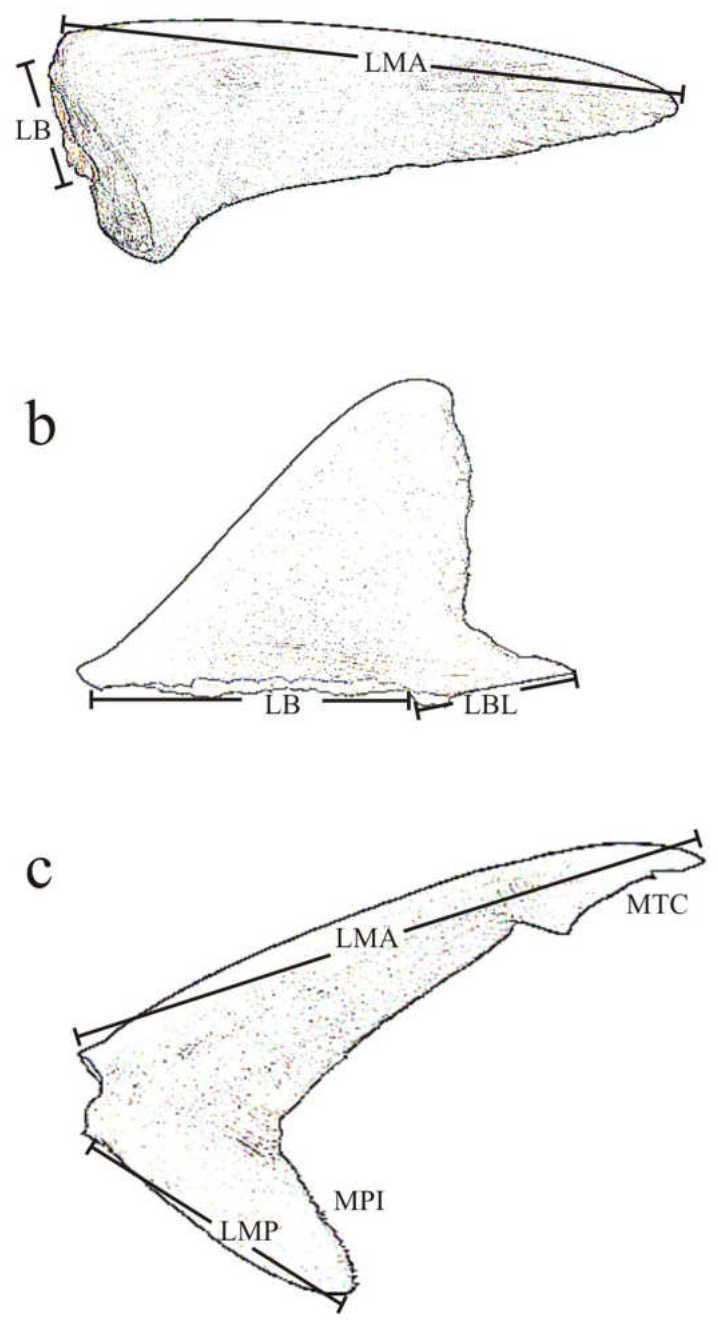

FIgURA 1. Medidas morfométricas utilizadas para cada tipo de aletas descritas y las siglas de los nombres de cada una. (a) Aleta pectoral: $\mathrm{LMA}=$ longitud margen anterior, $\mathrm{LB}=$ longitud base; (b) Primera aleta dorsal: $\mathrm{LB}=$ longitud base, LBL = longitud borde libre; (c) Aleta caudal, LMA = longitud margen anterior, LMP = longitud margen pre-ventral, MTC $=$ margen terminal caudal, MPI = margen pos-ventral inferior

FIGURE 1. Morphometric measurements used for each fin type described and the abbreviation of their names. (a) Pectoral fin: $\mathrm{LMA}=$ anterior margin length, $\mathrm{LB}=$ base length; (b) First dorsal fin: $\mathrm{LB}=$ base length, $\mathrm{LBL}=$ free border length; (c) Caudal fin: $\mathrm{LMA}=$ anterior margin length, $\mathrm{LMP}=$ pre-ventral margin length, $\mathrm{MTC}=$ caudal terminal length, MPI = post-ventral inferior margin. 
Identificación morfológica de aletas de tiburones: HERNANDEZ, S. ET AL.

CAudAL: La aleta tiene una notoria forma de media luna (Figura 11). El lóbulo superior es levemente más largo que el inferior (Tabla I). Es gris oscuro y no presenta una mancha blanca en la región debajo de la quilla. El margen terminal del lóbulo superior es recto (Figura 21). El margen post-ventral inferior es de forma convexa y contextura fina, con la presencia de notorias hilachas. Estas últimas, también se observan en el margen terminal del lóbulo superior. Presenta dos quillas laterales a cada lado.
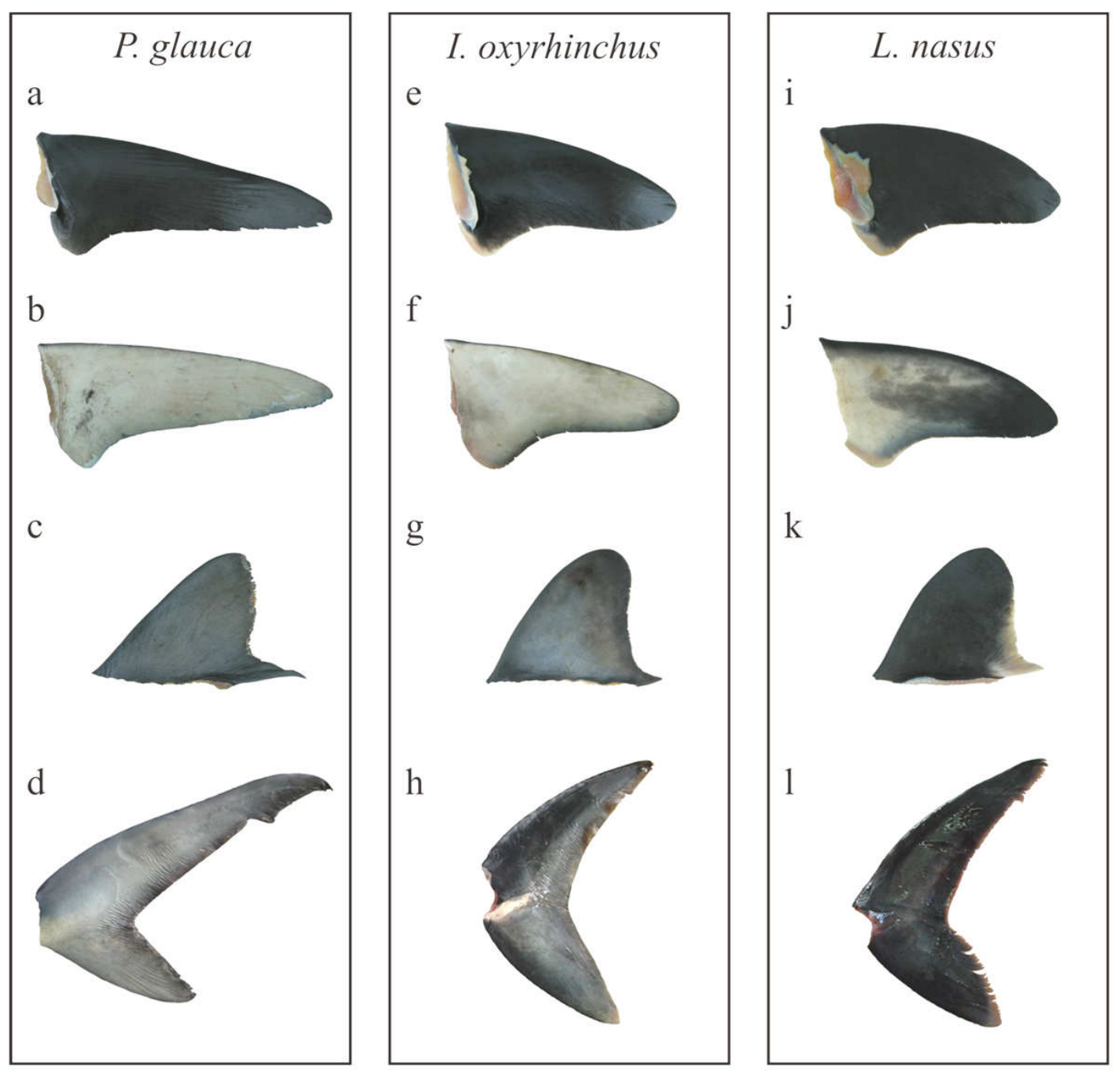

Figura 2. Fotografías de las aleta de los tiburones P. glauca (a-d), I. oxyrhinchus (e-h) y L. nasus (i-l). Aleta pectoral vista dorsal $(a, e, i)$, aleta pectoral vista ventral $(b, f, j)$, primera aleta dorsal $(c, g, k)$, y aleta caudal $(d, h, l)$.

Figure 2. Photographies of fins of the sharks P. glauca (a-d), I. oxyrhinchus (e-h), and L. nasus (i-1). Pectoral fin in dorsal view (a, e, i), pectoral fin in ventral view (b, f, j), first dorsal fin (c, g, k), and caudal fin (d, h, l). 
Gayana 73(1), 2009

\section{CLAVES TAXONOMICAS}

A continuación se presenta una clave taxonómica para cada tipo de aleta (pectoral, primera dorsal y caudal) que permiten identificar a las especies $P$. glauca, I. oxyrinchus y L. nasus.

PECTORAL

1a. Largo del margen anterior más de tres veces el de la base de la aleta $\quad P$ glauca

1b. Largo del margen anterior menos de tres veces el de la base de la aleta 2

2a. Lado ventral completamente blanco con bordes laterales levemente oscurecidos I. oxyrhinchus

2b. Lado ventral con un patrón negruzco característico desde el extremo de la aleta hasta el margen posterior interno

L. nasus

PRIMERA DORSAL

1a. Margen anterior recto y posterior curvado en forma de "V" P. glauca

1b. Margen anterior curvado y posterior levemente convexo. 2

2a. Extremo del borde libre azul oscuro I. oxyrinchus

$\begin{array}{ll}\text { 2b. Extremo del borde libre blanco } & \text { L. nasus }\end{array}$

Caudal

1a. Sin quillas laterales

P. glauca

1b. Presencia de quilla lateral

2

2a. Presenta una quilla lateral

I. oxyrinchus

2a. Presenta dos quillas laterales

L. nasus

\section{DISCUSION}

Los resultados demuestran que la forma y coloración son caracteres diagnósticos efectivos para la identificación de las aletas de los tiburones pelágicos más comercializados en Chile ( $P$. glauca, $I$. oxyrhinchus y L. nasus). La forma y la proporción de las mediciones morfométricas fueron los caracteres más importantes para identificar las aletas y distinguir entre las especies $P$. glauca y los lámnidos $I$. oxyrhinchus y L. nasus (Tabla I). Con respecto a estas especies de lámnidos, la forma general de las aletas no es un caracter diagnóstico para la identificación de las mismas. Sin embargo, se confirmó que el patrón de coloración podría ser utilizado para la identificación de las aletas entre ambas especies. De esta manera, en primera instancia I. oxyrhinchus y $L$. nasus podrían ser identificadas a partir de una serie de características relacionadas con el patrón de coloración. Se propone al patrón de coloración de la superficie ventral de la aleta pectoral, como suficiente para distinguir fácilmente entre ambas especies de lámnidos, e inclusive estimar tallas de captura a través del uso de sus proporciones corporales (Acuña et al. 2001; Hareide et al. 2007).

A partir del uso de los caracteres diagnósticos de las aletas propuestos en esta contribución es posible identificar a las tres especies pelágicas más importantes en el comercio de aletas (P. glauca, $I$. oxyrhinchus, y L. nasus) incluso si no se dispone del tronco, lo que podrá ser útil para mejorar la información especie-específica de las especies de tiburones capturados en Chile o por flotas chilenas en aguas internacionales. Tanto el Servicio Nacional de Pesca como el Aduanero de Chile, debería aumentar sus esfuerzos para registrar la composición, proporción y pesos de las aletas por especie, de manera de mejorar las estadísticas pesqueras y de exportación. Esta información es imperativa y esencial para llevar a cabo un plan de manejo nacional, enmarcado en el Plan de Acción Internacional para la Conservación y Ordenación de los Tiburones (FAO 1998), y cuyo Plan de Acción en Chile ya fue aprobado por la Subsecretaria de Pesca. 
Identificación morfológica de aletas de tiburones: HERNANDEZ, S. ET AL.

\section{AGRADECIMIENTOS}

Agradecemos a los comerciantes y colaboradores, Jorge Marín, Diego Alamos, Marcelo Rocco, Soledad Caro, Raquel Caro y Ciro Green, quienes permitieron la obtención de muestras (ejemplares completos y aletas).

\section{BIBLIOGRAFIA}

Acuña, E., Araya, M., Cid, L., Kong, I. \& Villarroel, J.C. 2001. Estudio biológico de tiburones (marrajo dentudo, azulejo y tiburón sardinero) en la zona norte y central de Chile. Informes Técnicos FIP. FIP/IT N ${ }^{\circ}$ 2000-23.112 pp.

AcuÑa, E., Villarroel J.c. \& Grau, R. 2002. Fauna íctica asociada a la pesquería del pez espada (Xiphias gladius Linnaeus). Gayana 66(2):263-267.

BARKer, M.J. \& SCHLUESSEL, V. 2005. Managing global shark fisheries: suggestions for prioritizing management strategies. Aquatic Conservation Marine and Freshwater Ecosystems 15(4):325-347.

Camhi, M., Fowler, S., Musick, J., Bräutigam, A. \& FordHAM, S. 1998. Sharks and their relatives: ecology and conservation. Occas. Pap. UICN Species Surviv. Comm. 20 pp.

Compagno, L. J. V. 2002. Sharks of the World. An Annotated and Illustrated Catalogue of Sharks Species Know to Date. Heterodontiformes, Lamniformes and Orectolobiformes. Food and Agriculture Organization, FAO Species Catalogue for Fisheries
Purposes Vol. 2, No 1. Rome. 269 pp.

Hareide, N.r., Carlson, J., Clarke, M., Clarke, S., Ellis, J., Fordham S., Fowler, S., Pinho, M., RaymaKers, C., Serena, F., Seret, B. \& Polti. S. 2007. European shark fisheries: a preliminary investigation into fisheries, conversion factors, trade products, markets and management measures. European Elasmobranch Association. 59 pp.

Hernandez, S., HaYe, P. \& ShivJ, M. 2008. Characterization of the pelagic shark fin trade in north central Chile by genetic identification and trader surveys. Journal of Fish Biology 73: 2293-2304.

Lamilla, J., Acuña, E., Araya, M., Kong, I., Oliva, M., Hernandez, S., Villarroel, J.c., Concha, F., Vögler, R., Pequeño, G. \& Mutschke, E. 2005. Lineamientos Básicos para Desarrollar el Plan de Acción Nacional de Tiburones. Informes Técnicos FIP. FIP/IT N ${ }^{\circ}$ 2004-18. Vol. 1. 125 pp.

Musick, J.A., Burgess, G., Cailliet, G., Cahm, M. \& Fordham, S. 2000. Management of sharks and their relatives (elasmobranchii). Fisheries. Vol. 25. No. 3.5 pp.

SERnAPESCA. 1996-2006. Anuarios Estadísticos de Pesca. Ministerio de Economía, Fomento y Reconstrucción, Chile.

Shivji, M., Clarke, S., Pank, M., Natanson, L., Kohler, N. \& Stanhope, M. 2002. Genetic identification of pelagic shark body parts for conservation and trade monitoring. Conservation Genetics 16(4):1036-1047.

Stevens, J.D., Bonfil, R., Dulvy, N.K. \& Walker, P.A. 2000. The effects of fishing on sharks, rays, and chimeras (chondrichthyans), and the implications for marine ecosystems. ICES Journal of Marine Science 57(3):476-494. 